\title{
Upgrading Plan Towards Multi-messenger Observation with LHAASO
}

\author{
Z. $\mathrm{CaO}^{1,2, a}$, M.J. Chen ${ }^{1}$, H.C. Li ${ }^{1}$, Z.G. Yao ${ }^{1}$, for LHAASO Collaboration \\ ${ }^{1}$ Institute of High Energy Physics \\ 19B, Yuquan St., Shijingshan, Beijing, China, 100049 \\ ${ }^{2}$ Chinese Academy of Science University \\ 19A, Yuquan St., Shijingshan, Beijing, China, 100049
}

\begin{abstract}
LHAASO is planning to enhance its sensitivity at energies around $100 \mathrm{GeV}$ by utilize MCP staffed 20" PMT in the Water Cherekove Detector Array. The effective area for gamma ray detection will reach to $1800 \mathrm{~m}^{2}$ and differential sensitivity to $0.2 \mathrm{CU}$ at $50 \mathrm{GeV}$. It will be the very useful survey detection for transient phenomena at $50 \mathrm{GeV}$ in the northern sky. LHAASO is expected to play an important role in the multimessenger observation with the upgrading.
\end{abstract}

\section{Introduction}

The first multi-messenger observation of gravitational wave (GW) event GW170817 by LIGO and VIRGO GW observatories together with many other Electro-magnetic (EM) wave observations, such as FERMI[1] and the multiwavelength campaign on the possible EM partner of the very high energy neutrino IceCube-170922A detected by IceCube experiment[2] are very significant progresses in astroparticle physics in the past year. They opened new windows for exploring the high energy phenomena in the universe. This, however, becomes a challenge to Large High Altitude Air Shower Observatory (LHAASO) experiment with its original proposal which is designed to target the high energy (above several hundred GeV) gamma ray sources and charged particles at even higher energies up to a few EeV. At energies lower than $300 \mathrm{GeV}$, the gamma ray detection sensitivity is not sufficient to detect those sources which is typically faint. In order to enhance the sensitivity below $100 \mathrm{GeV}$, we proposed to enlarge the sensitive area of the photo cathode of the water Cherenkov detector (WCD) in the LHAASO array. In this paper, we are going to briefly describe the LHAASO experiment and its WCD Array as well as the upgrading plan in particular in the second section, and the performance of WCDA in gamma ray astronomy with the the enhancement at low energy region in third section. The whole upgrading plan is summarized in the forth section.

\section{LHAASO/WCDA Experiment and the Upgrading Plan}

LHAASO is a multipurpose complex of EAS detection consisting of four major detector arrays[3], ie. 5195 scintillation counters (ED) and 1171 muon detectors (MD)

\footnotetext{
ae-mail: caozh@ihep.ac.cn
}

covering an area of $1.3 \mathrm{~km}^{2}, 78,000 \mathrm{~m}^{2}$ water Cherenkov detector (WCD) with 3120 gap-less detecting units, and 18 wide field of view Cherenkov telescopes watching over the sky above the whole complex with a coverage of 4608 square degree. As shown in Figure 1, the WCDA in the center of the array is divided into 3 components as 3 independent water pools, namely two smaller pools with the area of $150 m \times 150 \mathrm{~m}$ each and the larger one with the area of $300 m \times 110 m$.

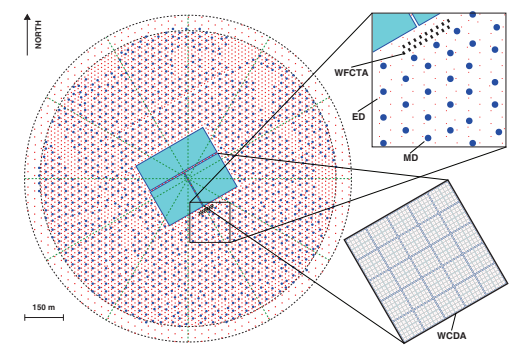

Figure 1. The layout of the scintillator counter (small dots) array, muon counter (big dots) array, water Cherenkov detector (rectangle in the center) array and 18 wide field of view Cherenkov telescopes (small rectangles) in the LHAASO complex of multidetector array of $1.3 \mathrm{~km}^{2}$.

The firstly built pool in south-west has 900 WCD units, $25 \mathrm{~m}^{2}$ each, equipped by a large (8") PMT for timing and a small (1.5") PMT for pulse size at the center of each unit $4 \mathrm{~m}$ beneath the water surface, and measures shower directions with a resolution better than $0.2^{\circ}$ above $10 \mathrm{TeV}$ and $1.0^{\circ}$ above $600 \mathrm{GeV}$. Only direct Cherenkov light generated by the shower particles is collected by the upwards watching PMTs. To suppress the cross talking effect and improve the timing resolution, black plastic divisions are 
installed between units. The Front End Electronics (FEE) of the large PMTs is designed to have the timing resolution of $0.5 \mathrm{~ns}$. The dynamic range of the detector is enlarged very much by using the small PMT. This enables the measurement of the detailed particle density distribution in the shower cores without significant saturation even for energetic showers up to $10 \mathrm{PeV}$ and achievement of the core location resolution better than $3 m$ over a wide energy range. This is designed for the identification of the primary particle species in the cosmic ray composition and spectrum measurements. It is also very useful in locating the shower inside the pool with minimal loss of good detected events. The pool is planned to be turned on for operation early 2019.

Low energy showers are small in terms of total number of particles that reach to the pools, therefore the total Cherenkov signal generated by those secondary particles in every detector unit is faint, even for units being near the cores of the showers. In order to enhance the gamma ray detecting sensitivity at low energies, enlarging the sensitive photo-cathode area of the PMT in the same size unit could be one effective way to catch the faint signals. LHAASO's upgrading plan is along with this approach, namely to replace the 8 " PMTs by 20 " PMTs in the rest two pools of $55,500 \mathrm{~m}^{2}$ in total. The customized design of the PMTs using multi-channel-plate (MCP) instead of the traditional dynodes enables good uniformity between PMTs as well as the Transit Time Spreads (TTS) less than $7 n s$, Cathode Transit Time Distribution (CTTD) less than $2 n s$ and long lifetime. The photo cathode is a factor of 6.25 larger than the 8 " tube, therefore the dynamic range is also shrunk by the same factor. In order to compensate the loss, a 3" PMT is installed beside the large PMT in each unit, read out only for the pulse size by a simplified version of FEE covering 4 orders of amplitudes in number of photo-electrons.

\section{Performances and Prospects for Gamma Ray Astronomy}

Gamma ray induced showers are different from showers induced by charged CR nuclei in terms of the hits distribution in the pool. In general, the later is more spread out than the former ones as shown in Figure 2, where two MC simulated events due to $1 \mathrm{TeV}$ gamma ray and $2 \mathrm{TeV}$ proton are compared with each other. Even more significantly, the CR events have many "hot spots", the populated hits, outside the core region, while the gamma ray events are much cleaner beyond some distance from the core. This enables us to identify the gamma events out of the CR background, nevertheless they are much more $\left(10^{4 \sim 5}\right)$ than gamma ray signals even if within a very small angular region defined by the point-spread-function (PSF) near the sources. By eliminating the events which have the most populated hit in the outer region, $45 \mathrm{~m}$ from the core, greater than certain number of photoelectrons, $N_{t h}$, the CR background will be suppressed to very low in the nearby region of sources. Making balancing between the elimination of the background CR events and the loss the gamma-

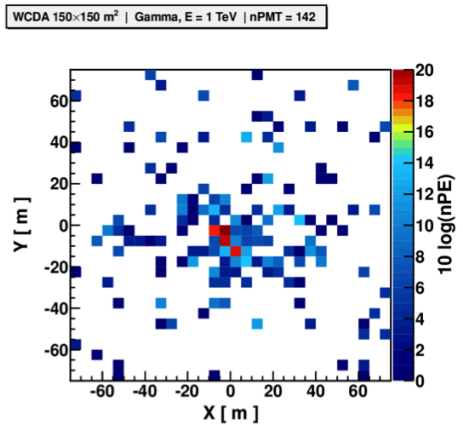

WCDA $150 \times 150 \mathrm{~m}^{2} \mid$ Proton, E = 2 TeV $\mid \mathrm{nPMT}=212$

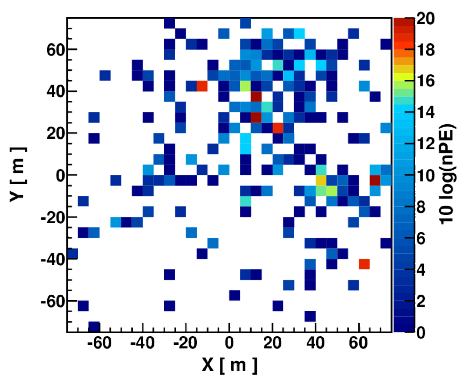

Figure 2. Two simulated events recorded by one of the pools of WCDA induced by $1 \mathrm{TeV}$ gamma ray (upper) and $2 \mathrm{TeV}$ proton (lower), respectively.

like signal events to maximize the sensitivity, it is found that $N_{t h}$ increases with the number of hits that are involved in the shower front fit, denoted as $N_{\text {fit }}$ which measures the shower energy, i.e. proportional to $N_{f i t} / \log N_{\text {fit }}$. Other parameters characterize the distribution of hits in the whole pool area, such as hit density in the outer region, are used in the identification of gamma ray events. This results an effective area for gamma ray detection of about $230 \mathrm{~m}^{2}$ at $50 \mathrm{GeV}$ and $30,000 \mathrm{~m}^{2}$ at $1 \mathrm{TeV}$, respectively, if all 3 pools were equipped by 8 " PMTs. The corresponding sensitivity of the gamma ray point-like source detection is plotted in Figure 3 as a function of gamma ray energy.

With this configuration, LHAASO has a survey power for discovering all sources that are brighter than $7 \mathrm{mini}$ crab unit above $1 \mathrm{TeV}$. Taking into account the wide field of view of $\sim 1 / 7$ of the entire sky and the constant exposure time of 24 hours, LHAASO is very significant in finding new sources. It is actually estimated that about 40 new AGNs[4] could be discovered within one year after it is fully operated. It is also expected that LHAASO will make a deeper survey inside our galaxy comparing with what has been done by HAWC experiment[5].

\subsection{Enhancement at Low Energy}

With the upgraded configuration using 20" PMTs in the other two pools, the effective area at energies below 300 $\mathrm{GeV}$ is significantly enlarged, i.e. reaches to $1,800 \mathrm{~m}^{2}$ at 


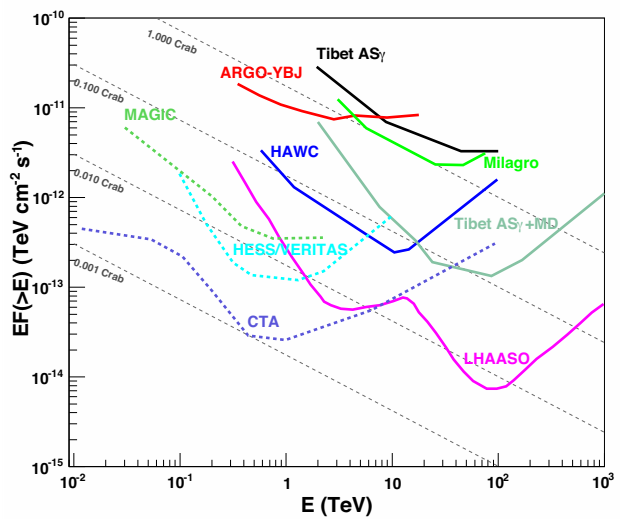

Figure 3. The integrated sensitivity of LHAASO (in pink) comparing with other experiments. The part of the curve below 10 $\mathrm{TeV}$ is the contribution of WCDA optimized with the cuts described in text.

$50 \mathrm{GeV}$ and $44,000 \mathrm{~m}^{2}$ at $1 \mathrm{TeV}$. The corresponding differential sensitivity around $50 \mathrm{GeV}$ is expected to be 0.2 crab unit per a quart decade of energy which is compatible with the space borne detector FERMI/LAT, as shown in Figure 4. The difference, however, is that the effective area is a factor of 1,800 larger than the later. This means that more than 1000 photons are expected to be recorded if the gamma ray burst event GRB090510 happened again in the field of view of LHAASO. In the event, FERMI/LAT recorded a single gamma photon at $95 \mathrm{GeV}[6]$. This opens a window for the multi-wavelength campaign in a much convenient way because of the clock-round operation of LHAASO. With any global alarm for transient phenomena, such as GBR, it is easy for LHAASO to recall the data in the window in which the alarm was ringing. Not only the status of the source at $T_{0}$ can be observed, but also it is in principle possible to find any pre-emission of gamma rays if there were.

With such a sensitivity, LHAASO will be a transient phenomenon finder as well. An alarm trigger algorithm is going to be operated to constantly watch all interested AGNs in LHAASO's FoV for any excess in various time windows, e.g. from few seconds to hours. It is useful for monitoring any AGN flare, e.g. if its emission level rises to be greater than $1 \mathrm{crab}$ unit within a hour, an alarm will be broadcasted to the whole community.

\subsection{Prospects for Multi-messenger Exploring}

Investigating sources with multi-messengers is very powerful in viewing of inside mechanism of high energy phenomena in the universe, particularly for possible common origins of the messengers, such us neutrinos, gamma rays, charged particles and gravitational waves. However, to identify the sources and verify the association, all corresponding detectors are required for sufficient sensitivities. As an example, we investigated the possible associ-

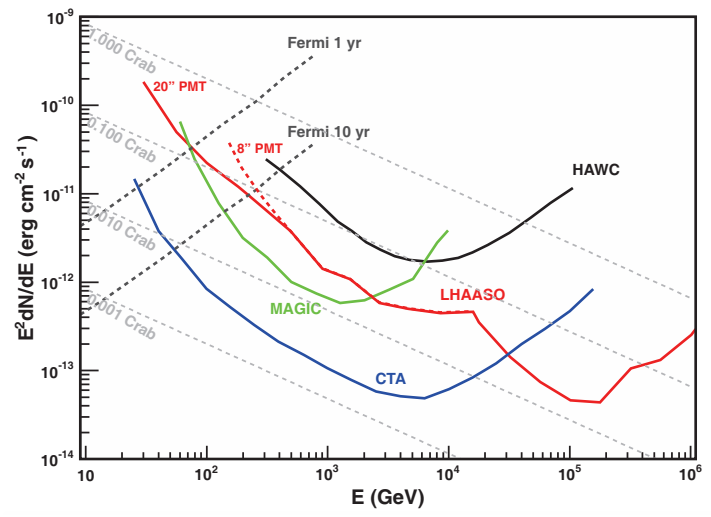

Figure 4. The differential sensitivity of LHAASO (in red) comparing with other experiments. Below $300 \mathrm{GeV}$, both estimates with 8" PMT configuration (dashed line) and 20" PMT configuration (solid line) are plotted. It is noticed that WCDA with 20" PMTs is almost as same sensitive as FERMI/LAT at $70 \mathrm{GeV}$.

ation between the ultra high energy muon neutrino event IC-170922A detected by IceCube experiment[2] and the blazer TXS $0506+056$ which had a faint flare within 20 days after the neutrino event in multiple wavelength bands, including ]X-ray (SWIFT), gamma ray (FERMI-LAT) and very high energy gamma ray (MAGIC). The SED of the blazer during the flare is reported in Ref. [2] and is quoted here in Figure 5 over a very wide energy range. Also shown in the figure, sensitivity curves of several experiments, including HAWK, HESS, VERITAS and upgraded LHAASO. According to this, LHAASO will play a significant role in such multi-messenger observation by covering an important energy range starting from as low as $30 \mathrm{GeV}$ to few hundred $\mathrm{TeV}$.

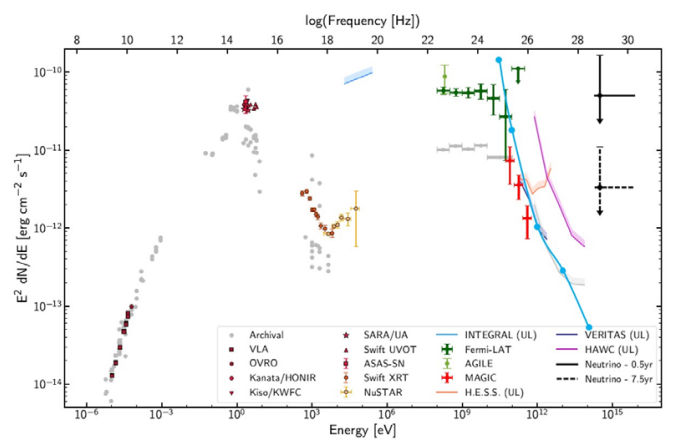

Figure 5. The SED of TXS $0506+056$ during the flare within 20 days after the neutrino event IC-170922A. It is reported in Ref. [2] including sensitivity curves of HAWC, HESS and VERITAS experiments. The upgraded LHAASO sensitivity is also plotted in the same figure (light blue) from $30 \mathrm{GeV}$ to $100 \mathrm{TeV}$. 


\section{Summary}

LHAASO has made its upgrading plan by replacing the $8 "$ PMTs in $70 \%$ of area of the central water Cherenkov detector with 20" PMTs. The first part, $30 \%$ of the total area of the detector, is under construction and going to be operated in early 2019 . The whole array, including the scintillator counter array and muon detector array and Cherenkov telescopes, will be built up by 2021. With the upgraded configuration, the gamma ray detecting sensitivity below $300 \mathrm{GeV}$ will be boosted to be about $0.2 \mathrm{crab}$ unit per quarter decades of energy around $50 \mathrm{GeV}$ and as same sensitive as FERMI at $70 \mathrm{GeV}$. LHAASO therefore will play a significant role in surveying for new sources brighter than 7 mini-crab-unit above $1 \mathrm{TeV}$ in the northern sky and monitoring for transient phenomena in its FoV of the size of $1 / 7$ sky at any moment. The LHAASO effective area of $1800 \mathrm{~m}^{2}$ around $50 \mathrm{GeV}$ is going to be useful tool in the multi-messenger observation involving ultra high energy neutrino or gravitational wave detections.

\section{5 acknowledgement}

We acknowledge the support to the author (CZ) for his attendance of the conference by the National Science Foundation of China under contract No. 11761141001.

\section{References}

[1] Abbott, B. P., et al. (LIGO and VIRGO Collaboration), PRL 119, 141101 (2017)

[2] Aartsen, M.G., et al., Science 361, eaat 1378 (2018) (arXiv:1807.08816)

[3] Z. Cao (for LHAASO Coll.), Chin.Phys. C34 249-252 (2010); H. He (for LHAASO Coll.), Radiation Detection Technology and Methods 2-7, (2018)

[4] Y. Zhao, et al., int.J.mode.Phys. D25 160, (2016)

[5] Abeysekara, A. U. et al., Astrophysical Journal 843 1, (2017)

[6] Ackermann, M et al., Science 343 6166, $42-47$ (2014) 